\title{
A Yukawa Interaction in Infinite Volume
}

\author{
JAMES GLimm*
}

Dept. of Mathematics, Mass. Institute of Technology, Cambridge, Mass.

ARTHUR $\mathrm{J}_{\mathrm{AFFE}}$ *ᄎ

Dept. of Physics, Harvard University, Cambridge, Mass.

Received June 18, 1968

Abstract. We prove the existence of the Heisenberg picture field operators for quantum fields with a Yukawa interaction. The interaction density

$$
g: \Psi^{+}(x) \Psi(x) \Phi(x):
$$

has a momentum cutoff on the Fermions, but no spatial form factor, and is thus translation invariant.

\section{\$1. Introduction}

We study the Heisenberg picture dynamics for a scalar Yukawa interaction $\Psi+\Psi \Phi$. The total Hamiltonian is not expected to be defined as an operator on Fock space, but it may be approximated by well defined cutoff Hamiltonians $H_{\varkappa V}$. In the approximate dynamics defined by the Hamiltonian $H_{\varkappa V}$, particles of energy $\varkappa$ or larger do not interact and particles located outside a bounded space region of volume $V$ do not interact. The operators $H_{\varkappa V}$ are positive and self adjoint $[6,7]$ and give rise to the Heisenberg picture dynamics

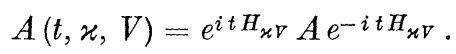

In two dimensional space-time the momentum cutoff may be removed if $V$ is held fixed and then the limit

$$
H_{V}=\lim _{x \rightarrow \infty} H_{x V}
$$

is a positive densely defined operator $[2,3]$.

In this paper we study the other cutoff and prove that a convergent subsequence

$$
A(t, \varkappa)=\lim _{j \rightarrow \infty} e^{i t H_{\varkappa V(j)} A e^{-i t H_{\varkappa V(j)}}}
$$

exists and is differentiable in $t$. The limit satisfies the differential equation

$$
\frac{d}{d t} A(t, \varkappa)=i\left[H_{\varkappa}, A\right](t, \varkappa)=i\left[H_{\varkappa}, A(t, \varkappa)\right] .
$$

* Supported in part by the National Science Foundation, NSF GP 7477.

$\star \star$ Supported in part by Air Force Office of Scientific Research. 
We note that $H_{\varkappa}$ has no meaning as an operator and the commutator in (1.4) is defined by limits:

$$
\left[H_{\varkappa}, A\right]=\lim _{V \rightarrow \infty}\left[H_{\varkappa V}, A\right] ;
$$

in fact $\left[H_{\varkappa V}, A\right]$ is independent of $V$ for large $V$ and for suitable $A$. The limit in (1.3) exists in the weak operator topology. For fixed $t$ the existence of a convergent subsequence (1.3) follows from very general compactness principles and is not the main difficulty. The essential point is the regularity in $t$ which permits convergence for all $t$.

We use the so-called "energy method" from Partial Differential Equations, which means that we make essential use of conserved or approximately conserved quantities to obtain convergence in (1.3). Let $R(t)$ be a positive operator depending on $t$. Then

If

$$
\begin{aligned}
& \frac{d}{d t}\left\langle e^{-i t H_{\varkappa V}} \varphi, R(-t) e^{-i t H_{\varkappa V}} \varphi\right\rangle \\
= & \left\langle e^{-i t H_{\varkappa V}} \varphi,-\left(\dot{R}(-t)+i\left[H_{\varkappa V}, R(-t)\right]\right) e^{-i t H_{\varkappa V}} \varphi\right\rangle .
\end{aligned}
$$

then $\left\|R(-t)^{1 / 2} e^{-i t H_{\varkappa V}} \varphi\right\|$ satisfies a differential inequality and

$$
\left\|R(-t)^{1 / 2} e^{-i t H_{\varkappa V}} \varphi\right\| \leqq \text { const. }\left\|R(0)^{1 / 2} \varphi\right\|
$$

where the constants depend on $T,|t| \leqq T$.

If the time dependence of $R(t)$ comes from the free field Heisenberg propagation,

then (1.7) simplifies to

$$
R(t)=e^{i t H_{0}} R(0) e^{-i t H_{0}},
$$

$$
i\left[H_{I \varkappa V}, R(-t)\right] \leqq \text { const. } R(-t)
$$

where $H_{I \varkappa V}$ is the interaction part of $H_{\varkappa V}=H_{0}+H_{I \varkappa V}$. We take $R(0)$ to be an operator which measures the local number of Bosons. The constants in (1.7) and (1.8) do not depend on $V$ and (1.8) will lead to volume independent estimates on the automorphism $A \rightarrow A(t, \varkappa, V)$.

The operators $A(t, x)$ will be defined on a dense domain $\mathscr{D}$ in the Fock space $\mathscr{F}$ of the bare particles. It would be desirable to have them act on a domain associated with physical particles. The physical Hilbert space can be constructed from a vacuum state and a vacuum state can be obtained by the method of [5], at least if the space cutoff were given by a periodic box.

We introduce our basic notation. $\Phi(x)$ will denote a scalar Boson field of mass $\mu_{0}>0$ and $\Pi$ is the conjugate momentum. $\Psi$ and $\Psi^{+}$are the Dirac field and its conjugate, which also have positive mass. Let $\varrho$ be 
a real function in the Schwartz space $\mathscr{S}$ and let

$$
\begin{aligned}
\Psi_{\varrho}(\boldsymbol{x}) & =\int \varrho(\boldsymbol{x}-\boldsymbol{y}) \Psi(\boldsymbol{y}) d \boldsymbol{y} \\
\Psi_{\varrho}^{+}(\boldsymbol{x}) & =\int \varrho(\boldsymbol{x}-\boldsymbol{y}) \Psi^{+}(\boldsymbol{y}) d \boldsymbol{y} \\
J(\boldsymbol{x}) & =\Psi_{\varrho}^{+}(\boldsymbol{x}) \Psi_{\varrho}(\boldsymbol{x}) \Phi(\boldsymbol{x}) .
\end{aligned}
$$

$\varrho$ introduces a momentum cutoff and replaces the $\varkappa$ used above; $\varrho$ is held fixed throughout the paper. Let $h$ be a positive function in $\mathscr{S}$ and let $\lambda$ be a positive number. We define

$$
\begin{aligned}
H_{I \lambda} & =\int h(\boldsymbol{x} / \lambda) J(\boldsymbol{x}) d x \\
H_{\lambda} & =H_{0}+H_{I \lambda} .
\end{aligned}
$$

Then $H_{I \lambda}$ is $H_{0}$-bounded and $H_{\lambda}$ is self adjoint and semibounded $[6,7]$. Let $U(t, \lambda)=\exp \left(-i t H_{\lambda}\right)$. The number $\lambda$ measures the volume cutoff and replaces the $V$ above.

Let

\section{§ 2. The Local Number of Bosons Operator}

$$
R(t)=I+\int \Phi^{-}(\boldsymbol{x}, t) \Phi^{+}(\boldsymbol{x}, t) \alpha(\boldsymbol{x}) d \boldsymbol{x},
$$

where $\Phi^{-}$is the creation part of the free field $\Phi$ and $\Phi^{+}$is the annihilation part of $\Phi$ and $\alpha$ is the positive integrable function

$$
\left(1+|x|^{2}\right)^{-2} \text {. }
$$

Computed in momentum space,

where

$$
R(t)=I+\int r(\boldsymbol{k}, \boldsymbol{l}, t) a^{*}(\boldsymbol{k}) a(\boldsymbol{l}) d \boldsymbol{k} d \boldsymbol{l}
$$

$$
r(\boldsymbol{k}, \boldsymbol{l}, t)=e^{i(\mu(\boldsymbol{k})-\mu(\boldsymbol{l})) t} \hat{\alpha}(\boldsymbol{k}-\boldsymbol{l}) \mu(\boldsymbol{k})^{-1 / 2} \mu(\boldsymbol{l})^{-1 / 2}
$$

and $\hat{\alpha}$ is the Fourier Transform of $\alpha$. One can check that $r$ is the kernel of a bounded operator on the one particle space. It follows that

$$
\left\|R(t)^{n} \varphi\right\| \leqq c^{n}\left\|N^{n} \varphi\right\|
$$

for some constant $c$. Thus any vector $\varphi$ with a finite number of particles is an analytic vector for $R$ and $R$ is essentially self adjoint on the domain consisting of these vectors. Also $R(t)$ is positive and $R(t)^{a}$ is essentially self adjoint on the same domain, for any real $a$.

We define

$$
\mathscr{D}=\prod_{-\infty<t<+\infty}^{\substack{\leq<n \\-1<+\infty}} \mathscr{D}\left(R(t)^{n}\right) .
$$

$\mathscr{D}$ will provide an invariant domain for the Heisenberg picture field operators. By our remarks above, $\mathscr{D}$ contains all vectors with a finite number of particles and so $\mathscr{D}$ is dense. 
In this section we derive our basic estimates showing that various operators can be bounded by powers of $R(t)$. In case the operators depend on the space cutoff $\lambda$, the bounds will be independent of $\lambda$. For example operators which are linear in the Boson field are dominated by $R(t)^{1 / 2}$.

Theorem 2.1. Let $\Omega \in \mathscr{D}\left(R(t)^{n+1 / 2}\right), n \geqq 0$ and let $f \in \mathscr{S}(x)$ be a Schwartz space function defined on configuration space. There is a continuous function $c(n, t)$ and a Schwartz space norm $|f|_{n}$ such that

$$
\left\|R(t)^{n} \Phi(f) \Omega\right\|+\left\|R(t)^{n} \Pi(f) \Omega\right\| \leqq c(n, t)\left\|R(t)^{n+1 / 2} \Omega\right\||f|_{n} .
$$

Proof. $\Phi^{ \pm}(\boldsymbol{x})=\Phi^{ \pm}(\boldsymbol{x}, 0)$ and $\Pi^{ \pm}(\boldsymbol{x})=\Pi^{ \pm}(\boldsymbol{x}, 0)$ may each be expressed in the form

$$
\int_{y_{0}=t} K^{ \pm}(\boldsymbol{x}-y) \Phi^{ \pm}(y) d \boldsymbol{y}
$$

where $K^{ \pm}(y) \in \mathcal{O}_{c}^{\prime}(\boldsymbol{y})$ for each fixed $y_{0}$ and depends continuously on $y_{0}$; $\mathcal{O}_{c}^{\prime}$ is the space of distributions for which an arbitrary derivative of the Fourier Transform is continuous and has at most polynomial growth. Thus $\Phi^{ \pm}(f)$ and $\Pi^{ \pm}(f)$ have the form

$$
\int_{y_{0}=t}\left(K^{ \pm} * f\right)(y) \Phi^{ \pm}(y) d \boldsymbol{y}
$$

where the convolution occurs in the space variables only, and since $K^{ \pm} \in \mathcal{O}_{c}^{\prime}, K^{ \pm} * f(\boldsymbol{x}, t) \in \mathscr{S}(\boldsymbol{x})$ for fixed $t$ and depends continuously on $f$ and $t$. Hence

for some $\mathscr{S}$ norm $|f|$.

$$
\begin{gathered}
\int\left|K^{ \pm} * f(\boldsymbol{x}, t)\right| d \boldsymbol{x} \leqq c_{0}(t)|f|, \\
\left|K^{ \pm} * f(\boldsymbol{x}, t)\right| \leqq c_{0}(t) \alpha(\boldsymbol{x})|f|
\end{gathered}
$$

To complete the proof in the case $n=0$, we may suppose that $f$ is real and that $\Omega$ is a vector with a finite number of particles with wave functions in $\mathscr{S}$. Then $\Omega \in \mathscr{D}\left(\Phi^{+}(x)\right)$ and $\Omega \in \mathscr{D}\left(\Pi^{+}(x)\right)$. One can verify that with a new norm $|f|$,

$$
\begin{aligned}
\|\Phi(f) \Omega\|^{2} \leqq & 4\left\|\Phi^{+}(f) \Omega\right\|^{2}+|f|^{2}\|\Omega\|^{2} \\
= & 4 \int_{x_{0}=y_{0}=t} \overline{K^{+} * f(x)} K^{+} * f(y)\left\langle\Phi^{+}(x) \Omega, \Phi^{+}(y) \Omega\right\rangle \\
& \cdot d \boldsymbol{x} d \boldsymbol{y}+|f|^{2}\|\Omega\|^{2} \\
\leqq & 4 \int_{x_{0}=y_{0}=t}\left|K^{+} * f(x) K^{+} * f(y)\right|\left(\left\|\Phi^{+}(x) \Omega\right\|^{2}+\left\|\Phi^{+}(y) \Omega\right\|^{2}\right) \\
& \cdot d \boldsymbol{x} d \boldsymbol{y}+|f|^{2}\|\Omega\|^{2} \\
\leqq & 8 c_{0}(t)^{2}|f|^{2} \int_{x_{0}=t} \alpha(x)\left\|\Phi^{+}(x) \Omega\right\|^{2} d \boldsymbol{x}+|f|^{2}\|\Omega\|^{2} \\
\leqq & 8 c_{0}(t)^{2}|f|^{2}\left\|R(t)^{1 / 2} \Omega\right\|^{2} .
\end{aligned}
$$

The estimates for $\Pi(f)$ are similar and the theorem is proved for $n=0$. 
The commutator $\left[\Phi^{\mp}(x, t), \Phi^{ \pm}(f)\right]$ or $\left[\Phi^{\mp}(x, t), \Pi^{ \pm}(f)\right]$ has the form

$$
K_{1} * K^{ \pm} * f
$$

where $K_{1} \in \mathcal{O}_{c}^{\prime}(x)$ and the convolutions are in the space variables only. Thus $\left[R(t), \Phi^{ \pm}(f)\right]$ and $\left[R(t), \Pi^{ \pm}(f)\right]$ have the form

$$
\int_{y_{0}=t} \alpha(\boldsymbol{y}) K_{1} * K^{ \pm} * f(y) \Phi^{ \pm}(y) d \boldsymbol{y}
$$

and the multiple commutators

and

$$
\left[R(t),\left[\ldots,\left[R(t), \Phi^{ \pm}(f)\right] \ldots\right]=(\operatorname{ad} R(t))^{n} \Phi^{ \pm}(f)\right.
$$

$$
\left[R(t),\left[\ldots,\left[R(t), \Pi^{ \pm}(f)\right] \ldots\right]=(\operatorname{ad} R(t))^{n} \Pi^{ \pm}(f)\right.
$$

have the form

$$
\int_{y_{0}=t} \alpha\left(K_{1} * \cdots * \alpha\left(K_{1} * K^{ \pm} * f\right) \ldots\right)(y) \Phi^{ \pm}(y) d \boldsymbol{y} .
$$

Now multiplication by $\alpha$ and convolution by $K_{1}$ are continuous operators on $\mathscr{S}(\boldsymbol{x})$ and so we have estimates similar to (2.6) and (2.7) for the coefficient of $\Phi^{ \pm}(y)$ in (2.9). As in the case $n=0$, this leads to a bound

$$
\begin{aligned}
\left\|(\operatorname{ad} R(t))^{n} \Phi(f) \Omega\right\|^{2} & +\left\|(\operatorname{ad} R(t))^{n} \Pi(f) \Omega\right\|^{2} \\
& \leqq c_{n}(t)|f|^{2}\left\|R(t)^{1 / 2} \Omega\right\|^{2}
\end{aligned}
$$

with a new norm $|f|$. The theorem now follows for arbitrary $n$, using (2.8) and (2.10).

Corollary 2.2. Let $A_{j}(\boldsymbol{x})$ denote one of the operators $\Phi(\boldsymbol{x}), \Pi(\boldsymbol{x}), \Psi(\boldsymbol{x})$ or $\Psi(\boldsymbol{x})^{+}, 1 \leqq j \leqq n$, and let $\Omega \in \mathscr{D}$. Then

$$
\left\langle\Omega, A_{1}\left(x_{1},\right) \ldots A_{n}\left(x_{n}\right) \Omega\right\rangle
$$

is in $\mathscr{S}^{\prime}$ as a function of $\boldsymbol{x}_{1}, \ldots, \boldsymbol{x}_{n}$.

Proof. By the nuclear theorem, we need only show that it is in $\mathscr{S}^{\prime}$ in each variable separately. But

$$
\begin{aligned}
& \left|\int f_{1}\left(\boldsymbol{x}_{1}\right) \ldots f_{n}\left(\boldsymbol{x}_{n}\right)\left\langle\Omega, A_{1}\left(\boldsymbol{x}_{1}\right) \ldots A_{n}\left(\boldsymbol{x}_{n}\right) \Omega\right\rangle d \boldsymbol{x}_{1} \ldots d \boldsymbol{x}_{n}\right| \\
= & \left|\left\langle\Omega, A_{1}\left(f_{1}\right) \ldots A_{n}\left(f_{n}\right) \Omega\right\rangle\right| \\
\leqq & \left\|A_{[n / 2]}\left(f_{[n / 2]}\right) \ldots A_{1}\left(f_{1}\right) \Omega\right\|\left\|\ldots A_{n}\left(f_{n}\right) \Omega\right\| .
\end{aligned}
$$

By Theorem 2.1, the quantity above is bounded by

$$
\left\|R(t)^{[n / 2]} \Omega\right\|\left\|R(t)^{[(n+1) / 2]} \Omega\right\| c(t) \prod_{i=1}^{n}\left|f_{i}\right|
$$

and the proof is complete.

Corollary 2.3. Let $A(\boldsymbol{x})$ denote $\Phi(\boldsymbol{x}), \Pi(\boldsymbol{x}), \Psi(\boldsymbol{x})$ or $\Psi^{+}(\boldsymbol{x})$ and let $j \geqq 0, n \geqq 0$. There is a continuous function $c(j, n, t)$ and $\mathscr{S}$-norms $|\cdot|_{j, n}$ such that

$$
\left\|R(t)^{n}\left(\operatorname{ad} H_{I \lambda}\right)^{j} A(f) \Omega\right\| \leqq c(j, n, t)|f|_{j, n}\left\|R(t)^{j+n+1 / 2} \Omega\right\|
$$

for $1 \leqq \lambda \leqq \infty$ and all $f \in \mathscr{S}$. 
Proof. One can compute directly that $(\operatorname{ad} R(t))^{m}\left(\operatorname{ad} H_{I \lambda}\right)^{j} A(f)$ is a sum of terms of the form

$$
\int s\left(\boldsymbol{x}_{1}, \ldots, \boldsymbol{x}_{k}\right)\left\langle\Omega, A_{1}\left(\boldsymbol{x}_{\mathbf{1}}\right) \ldots A_{k}\left(\boldsymbol{x}_{k}\right) \Omega\right\rangle d \boldsymbol{x}_{\mathbf{1}} \ldots d \boldsymbol{x}_{k}
$$

with $k \leqq j+1$. Also $s \in \mathscr{S}\left(\boldsymbol{x}_{1}, \ldots, \boldsymbol{x}_{k}\right)$ and $s$ depends continuously on $f$, so the corollary follows from Corollary 2.2 and (2.11).

Theorem 2.4. Let $n \geqq 0$. There is a continuous function $c(n, t)$ such that

$$
\left\|R(t)^{n}\left[R(t), H_{I \lambda}\right] \Omega\right\| \leqq c(n, t)\left\|R(t)^{n+1 / 2} \Omega\right\|
$$

for $1 \leqq \lambda \leqq \infty, \Omega \in \mathscr{D}\left(R(t)^{n+1 / 2}\right)$.

Proof. Let

$$
\begin{aligned}
r_{\lambda, n}(\boldsymbol{x}, t) & =\alpha(\boldsymbol{x}) \int \Delta^{(+)}(\boldsymbol{y}-x) \Psi_{\varrho}^{+}(\boldsymbol{y}) \Psi_{\varrho}(\boldsymbol{y}) h(\boldsymbol{y} / \lambda) d \boldsymbol{y} \\
r_{\lambda, n}(\boldsymbol{x}, t) & =\alpha(\boldsymbol{x})\left(\Delta^{(+)}(\cdot, 0) * r_{\lambda, n-1}\right)(\boldsymbol{x}, t) \\
A_{\lambda, n}(t) & =\int r_{\lambda, n}(\boldsymbol{x}, t)^{*} \Phi^{+}(\boldsymbol{x}, t) d \boldsymbol{x} \\
B_{\lambda, n}(t) & =\int \Phi^{-}(\boldsymbol{y}, t) r_{\lambda, n}(\boldsymbol{x}, t)^{*} r_{\lambda, n}(\boldsymbol{y}, t) \Phi^{+}(\boldsymbol{x}, t) d \boldsymbol{x} d \boldsymbol{y} \\
C_{\lambda, n}(t) & =\int r_{\lambda, n}(\boldsymbol{x}, t)^{*} r_{\lambda, n}(\boldsymbol{y}, t) i^{-1} \Delta^{(+)}(\boldsymbol{x}-\boldsymbol{y}, 0) d \boldsymbol{x} d \boldsymbol{y} .
\end{aligned}
$$

To prove the theorem it is sufficient to show that

$$
\left\|(\operatorname{ad} R(t))^{n} H_{I \lambda} \Omega\right\| \leqq c(n, t)\left\|R(t)^{1 / 2} \Omega\right\|
$$

for $n \geqq 1$. One can show that (ad $R(t))^{n} H_{I \lambda}$ is a linear combination of $A_{\lambda n}(t)$ and $A_{\lambda n}(t) *$ and that

$$
A_{\lambda, n}(t) A_{\lambda, n}(t)^{*}=B_{\lambda, n}(t)+C_{\lambda, n}(t)
$$

and so it is sufficient to show that

$$
\begin{gathered}
\left\|A_{\lambda, n}(t) \Omega\right\|^{2}+\left|\left\langle\Omega, B_{\lambda, n}(t) \Omega\right\rangle\right|+\left|\left\langle\Omega, C_{\lambda, n}(t) \Omega\right\rangle\right| \\
\leqq c(n, t)\left\|R(t)^{1 / 2} \Omega\right\|^{2} .
\end{gathered}
$$

We use the following lemma.

Lemma 2.5. Let $D$ be a monomial in the space derivatives of order $s$. There are continuous functions $c_{2}(s, n, t)$ and $c_{3}(n, t)$ such that

$$
\begin{aligned}
&\left\|D r_{\lambda, n}(\boldsymbol{x}, t)\right\| \leqq c_{2}(s, n, t) \alpha(\boldsymbol{x}), \\
&\left\|C_{\lambda, n}(t)\right\| \leqq c_{3}(n, t),
\end{aligned}
$$

and $c_{2}$ and $c_{3}$ are independent of $\lambda, \lambda \in[1, \infty]$.

From the lemma we have

$$
\begin{aligned}
\left\|A_{\lambda, n}(t) \Omega\right\|^{2} & =\left(\left\|\int r_{\lambda, n}(\boldsymbol{x}, t)^{*} \Phi^{+}(\boldsymbol{x}, t) d \boldsymbol{x} \Omega\right\|\right)^{2} \\
& \leqq\left(\int\left\|r_{\lambda, n}(\boldsymbol{x}, t)\right\|\left\|\Phi^{+}(\boldsymbol{x}, t) \Omega\right\| d \boldsymbol{x}\right)^{2} \\
& \leqq \int\left\|r_{\lambda, n}(\boldsymbol{x}, t)\right\| d \boldsymbol{x} \int\left\|r_{\lambda, n}(\boldsymbol{x}, t)\right\|\left\|\Phi^{+}(\boldsymbol{x}, t) \Omega\right\|^{2} d \boldsymbol{x} \\
& \leqq c_{2}(0, n, t)^{2} \int \alpha(\boldsymbol{x}) d \boldsymbol{x}\left\|R(t)^{1 / 2} \Omega\right\|^{2}
\end{aligned}
$$


if $\Omega$ is a vector with a finite number of particles and wave functions in $\mathscr{S}$, so that $\Omega \in \mathscr{D}\left(\Phi^{+}(\boldsymbol{x}, t)\right)$. By continuity the inequality extends to all $\Omega$ in $\mathscr{D}\left(R(t)^{1 / 2}\right)$ and similarly

$$
\left|\left\langle\Omega, B_{\lambda, n}(t) \Omega\right\rangle\right| \leqq c_{2}(0, n, t)^{2} \int \alpha(x) d x\left\|R(t)^{1 / 2} \Omega\right\|^{2},
$$

and then the theorem is proved.

Proof of Lemma 2.5. We have

$$
\begin{aligned}
\Psi_{\varrho}(\boldsymbol{x}) & =\int \varrho(\boldsymbol{x}-\boldsymbol{y}) \Psi(\boldsymbol{y}) d \boldsymbol{y} \\
D \Psi_{\varrho}(\boldsymbol{x}) & =\int(D \varrho)(\boldsymbol{x}-\boldsymbol{y}) \Psi(\boldsymbol{y}) d \boldsymbol{y}
\end{aligned}
$$

and it follows from the anticommutation relations that $\Psi_{\varrho}(x)$ and $D \Psi_{\varrho}(x)$ are bounded operators:

$$
\begin{gathered}
\left\|\Psi_{\varrho}(x)\right\| \leqq\|\varrho\|_{2} \\
\left\|D \Psi_{\varrho}(x)\right\| \leqq\|D \varrho\|_{2} .
\end{gathered}
$$

The same argument applies to $\Psi_{\varrho}^{+}(\boldsymbol{x})$ and $D \Psi_{\varrho}^{+}(\boldsymbol{x})$ and so

where

$$
\left\|D J_{\lambda}(x)\right\| \leqq c
$$

$$
J_{\lambda}(\boldsymbol{x})=\Psi_{\varrho}^{+}(\boldsymbol{x}) \Psi_{\varrho}(\boldsymbol{x}) h(\boldsymbol{x} / \lambda)
$$

and $c$ is a constant independent of $x$ and of $\lambda \geqq 1$. The distribution $\Delta^{(+)}(x)$ is a $C^{\infty}$ function except on the cone $|x|=t$, and from the explicit formulae for $\Delta^{(+)},[1]$, we can write

$$
\Delta^{(+)}(\boldsymbol{x}, t)=\theta_{\mathbf{1}}(\boldsymbol{x}, t)+\theta_{\mathbf{2}}(\boldsymbol{x}, t)
$$

where $\theta_{2}(\cdot, t) \in \mathscr{S}(\boldsymbol{x})$ and depends continuously on $t$ and

$$
\theta_{1}(x)=\sum_{\mu} D_{\mu} \eta_{\mu}(x)
$$

Here $D_{\mu}$ is a monomial in the space derivatives and $\eta_{\mu}$ is a continuous function with compact support in $\boldsymbol{x}$ for each fixed $t$, and the sum over $\mu$ is finite. Thus

$$
\begin{aligned}
\left\|r_{\lambda, l}(\boldsymbol{x}, t)\right\| \leqq & \alpha(\boldsymbol{x}) \sum_{\mu} \int\left|\eta_{\mu}(\boldsymbol{y}-x)\right|\left\|D_{\mu} J_{\lambda}(\boldsymbol{y})\right\| d \boldsymbol{y} \\
& +\alpha(\boldsymbol{x}) \int\left|\theta_{2}(\boldsymbol{y}-x)\right|\left\|J_{\lambda}(\boldsymbol{y})\right\| d \boldsymbol{y} \\
= & c_{2}(0,1, t) \alpha(\boldsymbol{x})
\end{aligned}
$$

as required. The derivatives $D r_{\lambda, l}(\boldsymbol{x}, t)$ are estimated similarly. Some of the derivatives are applied to $\alpha$, and by our explicit definition of $\alpha$,

$$
\left|D_{\nu} \alpha(x)\right| \leqq c(v) \alpha(x) \text {. }
$$

The remaining derivatives are applied to $J_{\lambda}$ and do not cause trouble because $\left\|D_{v} J_{\lambda}(\boldsymbol{y})\right\|$ is bounded uniformly in $y$ and in $\lambda \geqq 1$. In the same fashion the bound

$$
\left\|D r_{\lambda, n}(\boldsymbol{x}, t)\right\| \leqq c_{2}(s, n, t) \alpha(\boldsymbol{x})
$$


is proved using our inductive definition of $r_{\lambda, n}$. Finally

$$
\begin{aligned}
\left\|C_{\lambda, n}(t)\right\| \leqq & \sum_{\mu} \int\left\|r_{\lambda, n}(\boldsymbol{x}, t)\right\|\left\|D_{\mu} r_{\lambda, n}(\boldsymbol{y}, t)\right\|\left|\eta_{\mu}(\boldsymbol{x}-\boldsymbol{y}, 0)\right| d \boldsymbol{x} d \boldsymbol{y} \\
& +\int\left\|r_{\lambda, n}(\boldsymbol{x}, t)\right\|\left\|r_{\lambda, n}(\boldsymbol{y}, t)\right\|\left|\theta_{2}(\boldsymbol{x}-\boldsymbol{y}, 0)\right| d \boldsymbol{x} d \boldsymbol{y} \\
\leqq & \sum_{\mu} c_{2}(0, n, t) c_{2}(|\mu|, n, t)\left(\int \alpha(\boldsymbol{x}) d \boldsymbol{x}\right)^{2}\left\|\eta_{\mu}(\cdot, 0)\right\|_{\infty} \\
& +c_{2}(0, n, t)^{2}\left(\int \alpha(\boldsymbol{x}) d \boldsymbol{x}\right)^{2}\left\|\theta_{2}(\cdot, 0)\right\|_{\infty}=c_{3}(n, t) .
\end{aligned}
$$

Corollary 2.6. There is a continuous function $c(n, t)$ independent of $\lambda$ such that

$$
\left\|R(-t)^{n} U(t, \lambda) \Omega\right\| \leqq c(n, t)\left\|R(0)^{n} \Omega\right\| \text {. }
$$

Proof. Let $\Omega$ be a state with a finite number of particles. Then $U(t, \lambda) \Omega \in \mathscr{D}\left(N^{n}\right) \subset \mathscr{D}\left(R(-t)^{n}\right)$ and

$$
\begin{aligned}
& \frac{d}{d t}\left\langle U(t, \lambda) \Omega, R(-t)^{n} U(t, \lambda) \Omega\right\rangle \\
= & \left\langle U(t, \lambda) \Omega, i\left[H_{I \lambda}, R(-t)^{n}\right] U(t, \lambda) \Omega\right\rangle \\
\leqq & \sum_{j=1}^{n} c(j, t)\left\langle U(t, \lambda) \Omega, R(-t)^{j} U(t, \lambda) \Omega\right\rangle \\
\leqq & c_{1}(n, t)\left\langle U(t, \lambda) \Omega, R(-t)^{n} U(t, \lambda) \Omega\right\rangle .
\end{aligned}
$$

The corollary follows by an integration over $t$.

\section{\$ 3. The Heisenberg Picture Fields}

We define the Heisenberg picture field $A_{H}(\boldsymbol{x}, t)$ as the limit of a weakly convergent sequence

$$
\begin{aligned}
A_{H}(\boldsymbol{x}, t) & =\lim _{j \rightarrow \infty} U\left(t, \lambda_{j}\right)^{*} A(\boldsymbol{x}) U\left(t, \lambda_{j}\right) \\
A(\boldsymbol{x}) & =\Phi(\boldsymbol{x}), \Pi(\boldsymbol{x}), \Psi(\boldsymbol{x}) \text { or } \Psi^{+}(\boldsymbol{x}) .
\end{aligned}
$$

$A_{H}(\boldsymbol{x}, t)$ is defined for each fixed $t$ as an operator valued distribution in $\mathscr{S}^{\prime}(\boldsymbol{x})$; if $f \in \mathscr{S}(\boldsymbol{x})$ then

$$
A_{H}(f, t)=\int A_{H}(\boldsymbol{x}, t) f(\boldsymbol{x}) d \boldsymbol{x}
$$

is an unbounded operator with domain $\mathscr{D}$ and

$$
A_{H}(f, t) \mathscr{D} \subset \mathscr{D} \text {. }
$$

$A_{H}(f, t)$ is smooth as a function of $t$ (strong derivatives) and satisfies the differential equation (1.4).

Theorem 3.1. There is a sequence $\lambda_{j} \rightarrow \infty$ such that for all $f \in S(x)$, $\Omega \in \mathscr{D}, \varphi \in \mathscr{F}$ and for all $t$ the limit

$$
\left\langle\varphi, A_{H}(f, t) \Omega\right\rangle=\lim _{j \rightarrow \infty}\left\langle\varphi, U\left(t, \lambda_{j}\right)^{*} A(f) U\left(t, \lambda_{j}\right) \Omega\right\rangle
$$

exists and defines $A_{H}(f, t)$ as an operator. 
Proof. For fixed $f, \Omega, \varphi$ and $t$ we have the uniform estimate

$$
\begin{aligned}
\left\|U\left(t, \lambda_{j}\right)^{*} A(f) U\left(t, \lambda_{j}\right) \Omega\right\| & =\left\|A(f) U\left(t, \lambda_{j}\right) \Omega\right\| \\
& \leqq c(t)|f|\left\|R(-t)^{1 / 2} U\left(t, \lambda_{j}\right) \Omega\right\| \\
& \leqq c(t)|f|\left\|R(0)^{1 / 2} \Omega\right\|
\end{aligned}
$$

from Theorem 2.1 and Corollary 2.6 and by weak compactness there is a sequence $\lambda_{j}$ such that the limit (3.4) exists. By the diagonal process we achieve the same result for a countable dense set of $f$ 's, $\Omega$ 's, $\varphi$ 's and t's and the $\Omega$ 's are dense in $\mathscr{D}\left(R(0)^{1 / 2}\right)$ in the norm $\left\|R(0)^{1 / 2} \Omega\right\|$. By continuity and the uniform estimate (3.5), we have convergence for all $f, \Omega$ and $\varphi$. Using the Ascoli theorem, we get convergence for all $t$. To apply Ascoli's theorem we need the following uniform estimate on the time derivatives:

$$
\begin{aligned}
& \left\|(d \mid d t) U\left(t, \lambda_{j}\right)^{*} A(f) U\left(t, \lambda_{j}\right) \Omega\right\| \\
= & \left\|U\left(t, \lambda_{j}\right)^{*}\left[H_{\lambda_{j}}, A(f)\right] U\left(t, \lambda_{j}\right) \Omega\right\| \\
= & \left\|\left[H_{\lambda_{j}}, A(f)\right] U\left(t, \lambda_{j}\right) \Omega\right\| \\
\leqq & c(t)|f|\left\|R(-t)^{1 / 2} U\left(t, \lambda_{j}\right) \Omega\right\| \\
\leqq & c_{\mathbf{1}}(t)|f|\left\|R(0)^{1 / 2} \Omega\right\| .
\end{aligned}
$$

Our estimates also imply

$$
\left|\left\langle\varphi, A_{H}(f, t) \Omega\right\rangle\right| \leqq c(t)|f|\|\varphi\|\left\|R(0)^{1 / 2} \Omega\right\|
$$

and so $A_{H}(f, t)$ is an operator with the domain $\mathscr{D}$.

Corollary 3.2. For $\Omega \in \mathscr{D}$ we have

$$
\left\|R(t)^{n} A_{H}(f, s) \Omega\right\| \leqq c(n, t, s)|f|_{n}\left\|R(t)^{n+1} \Omega\right\|
$$

for some continuous function $c$ and some norm $|f|_{n}$. In particular (3.3) holds.

Proof.

$$
\begin{aligned}
& \left\|R(t)^{n} U\left(s, \lambda_{j}\right)^{*} A(f) U\left(s, \lambda_{j}\right) \Omega\right\| \\
\leqq & c_{1}(n, t, s)\left\|R(t-s)^{n} A(f) U\left(s, \lambda_{j}\right) \Omega\right\| \\
\leqq & c_{2}(n, t, s)|f|_{n}\left\|R(t-s)^{n+1} U\left(s, \lambda_{j}\right) \Omega\right\| \\
\leqq & c_{3}(n, t, s)|f|_{n}\left\|R(t)^{n+1} \Omega\right\|
\end{aligned}
$$

by Theorem 2.1 and Corollary 2.6.

Corollary 3.3. $A_{H}(f, t)$ is $C^{\infty}$ as a function of $t$ for $\Omega \in \mathscr{D}$,

$$
\frac{d}{d t} A_{H}(f, t) \Omega=i\left[H, A_{H}(f, t)\right] \Omega
$$

where we define the commutator by the limits

$$
\begin{aligned}
& \left\langle\varphi,\left[H, A_{H}(f, t)\right] \Omega\right\rangle \\
= & \lim _{j \rightarrow \infty}\left\langle\varphi,\left[H_{\lambda_{j}}, U\left(t, \lambda_{j}\right)^{*} A(f) U\left(t, \lambda_{j}\right)\right] \Omega\right\rangle \\
= & \lim _{j \rightarrow \infty}\left\langle\varphi, U\left(t, \lambda_{j}\right)^{*}\left[H_{\lambda_{j}}, A(f)\right] U\left(t, \lambda_{j}\right) \Omega\right\rangle .
\end{aligned}
$$

In particular the limits in (3.7) exist.

2 Commun. math. Phys., Vol.11 
Proof. Using Corollary 2.3, we have the uniform estimate

$$
\left\|(d / d t)^{n} U\left(t, \lambda_{j}\right)^{*} A(f) U\left(t, \lambda_{j}\right) \Omega\right\| \leqq c(n, t)|f|_{n}\left\|R(0)^{n+1 / 2} \Omega\right\|
$$

as in (3.6). This implies that the function (3.4) is $C^{\infty}$ in $t$ and

$$
\left|\left\langle\varphi,(d \mid d t)^{n} A_{H}(f, t) \Omega\right\rangle\right| \leqq c(n, t)|f|_{n}\|\varphi\|\left\|R(0)^{n+1 / 2} \Omega\right\| \text {. }
$$

As in the proof of Theorem 2.1, we find a subsequence $\lambda_{j_{i}}$ for which the limits (3.8) exist. Any such limit will satisfy the Eq. (3.7) and since the left side of (3.7) is already known to exist and is uniquely determined, all subsequences $\lambda_{j_{i}}$ in (3.8) have the same limit. Thus the full sequence converges in (3.8).

We remark that for $\Omega \in \mathscr{D}$ the expectation value

$$
\left\langle\Omega, A_{H}\left(x_{1}, t_{1}\right) \ldots A_{H}\left(x_{n}, t_{n}\right) \Omega\right\rangle
$$

is in $\mathscr{S}^{\prime}$ as a function of the space variables for fixed $t_{1}, \ldots, t_{n}$. Also $(d / d t)^{n} A_{H}(f, t) \mathscr{D} \subset \mathscr{D}$. A theory invariant under space translations could be obtained by introducing a translated local number of Bosons operator $R(\boldsymbol{a}, t)$ and redefining $\mathscr{D}$ as $\bigcap_{\boldsymbol{a}, t, n} \mathscr{D}\left(R(\boldsymbol{a}, t)^{n}\right)$. It would be desirable to show that the limit (3.4) converges strongly on the domain $\mathscr{D}$. Strong convergence would imply the canonical commutation and anticommutation relations for the fields $A_{H}(x, t)$.

\section{Bibliography}

1. Bogoliduov, N., and D. Shrrkov: Introduction to the theory of quantized fields. New York: Interscience Publ. 1959.

2. Glimm, J.: Yukawa coupling of quantum fields in two dimensions, I. Commun. Math. Phys. 5, 343-386 (1967).

3. - Yukawa coupling of quantum fields in two dimensions, II. Commun. Math. Phys. 6, 120-132 (1967).

4. - and A. JAFFE: A $\lambda \phi^{4}$ quantum field theory without cutoffs. Phys. Rev. (To appear.)

5. JAFFE, A., and R. Powers: Infinite volume limit of a $\lambda \phi^{4}$ field theory. Commun. Math. Phys. 7, 218 (1968).

6. Kato, Y.: Some converging examples of perturbation series in quantum field theory. Prog. Theor. Phys. 26, 99 (1961).

7. LANFORD, O.: Construction of quantum fields interacting by a cutoff Yukawa coupling. Princeton University Thesis, 1966.

J. GLIMM

Department of Mathematics

Mass. Institute of Technology

Cambridge, Mass. 02139, USA

\section{A. JAFFE}

Department of Physics

Harvard University

Cambridge, Mass. 02139, USA 